\title{
Identification of Common Genes Refers to Colorectal Carcinogenesis with Paired Cancer and Noncancer Samples
}

\author{
Lihua Zhang $\mathbb{D}^{1,2}$ Yonglong Yang $\mathbb{D}^{1,}{ }^{3}$ Lin Cheng, ${ }^{4}$ Yu Cheng $\mathbb{D}^{1,2}$ Hong-Hao Zhou ${ }^{\mathbb{D}},{ }^{1,2}$ \\ and Zhi-Rong Tan $\mathbb{1}^{1,2}$ \\ ${ }^{1}$ Department of Clinical Pharmacology, Xiangya Hospital, Central South University, Changsha 410008, China \\ ${ }^{2}$ Institute of Clinical Pharmacology, Central South University, Hunan Key Laboratory of Pharmacogenetics, Changsha 410078, China \\ ${ }^{3}$ Haikou People's Hospital and Affiliated Haikou Hospital of Xiangya Medical School, Central South University, Haikou, \\ Hainan 570311, China \\ ${ }^{4}$ State Key Laboratory of Ophthalmology, Zhongshan Ophthalmic Center, Sun Yat-sen University, 54 South Xianlie Road, \\ Guangzhou, Guangdong, China
}

Correspondence should be addressed to Hong-Hao Zhou; hhzhou2003@163.com and Zhi-Rong Tan; zrtan800820@163.com

Received 21 June 2017; Accepted 16 October 2017; Published 30 January 2018

Academic Editor: Stamatios E. Theocharis

Copyright (C) 2018 Lihua Zhang et al. This is an open access article distributed under the Creative Commons Attribution License, which permits unrestricted use, distribution, and reproduction in any medium, provided the original work is properly cited.

\begin{abstract}
Colorectal cancer is a malignant tumor which harmed human beings' health. The aim of this study was to explore common biomarkers associated with colorectal carcinogenesis in paired cancer and noncancer samples. At first, fifty-nine pairs of colorectal cancer and noncancer samples from three gene expression datasets were collected and analyzed. Then, 181 upregulation and 282 downregulation common differential expression genes (DEGs) were found. Next, functional annotation was performed in the DAVID database with the DEGs. Finally, real-time polymerase chain reaction (PCR) assay was conducted to verify the analyses in sixteen colorectal cancer and individual-matched adjacent mucosa samples. Real-time PCR showed that MCM2, RNASEH2A, and TOP2A were upregulated in colorectal cancer compared with adjacent mucosa samples $(M C M 2, P<0.001 ; R N A S E H 2 A, P<0.001$; TOP2A, $P=0.001)$. These suggested that $463 \mathrm{DEGs}$ might contribute to colorectal carcinogenesis.
\end{abstract}

\section{Introduction}

Colorectal cancer is one of the most commonly diagnosed cancers worldwide, with approximately 1.4 million cases and 693,900 deaths in 2012 [1]. Some risk factors may lead to colorectal cancer such as family history $[2,3]$, inflammatory bowel disease [4], and smoking $[5,6]$. In the molecular level, colorectal cancer is a heterogeneous disease. Mutations, epigenetic changes, and expression differences of multiple genes are well-known colorectal cancer contributors. However, the underlying molecular mechanism of colorectal carcinogenesis has not been fully understood yet.

More than twenty years ago, complementary DNA microarray has been used to analyze the gene expression patterns in human cancer [7]. With the development of technology, more and more expression profile platforms and researches on colorectal cancer have been released in recent years. Currently, a large number of expression profile datasets were uploaded and shared publically on several public databases, and Gene Expression Omnibus (GEO, https://www.ncbi.nlm.nih.gov/geo) and ArrayExpress database (http://www.ebi.ac.uk/arrayexpress) were included. The public shared data of the databases could benefit the researchers greatly in finding interesting research targets or verify their ideas easily.

In this study, to further understand the mechanism of colorectal carcinogenesis from the gene expression level, we searched the GEO database for paired colorectal cancer and noncancer samples. Fifty-nine paired samples from GSE21510, GSE23878, and GSE32323 were selected in total. The DEGs were screened from three datasets, and 463 common DEGs (181 upregulation and 282 downregulation genes) were detected and sent to the DAVID database (https://david.ncifcrf.gov) for functional annotation. Three 
upregulated genes were chosen to verify the analysis results in sixteen pairs of colorectal cancer and adjacent mucosa tissues. Real-time PCR results showed that MCM2, RNA$S E H 2 A$, and TOP2A were associated with colorectal carcinogenesis.

\section{Materials and Methods}

2.1. Microarray Data. We searched the NCBI-GEO database with the following keywords: Human Genome U133 Plus 2.0, colorectal cancer, and normal (Human Genome U133 Plus 2.0 Array is a platform of Affymetrix). Fifty-nine paired colorectal cancer and noncancerous samples were selected from three gene expression datasets (twenty-three pairs in GSE21510 from Japan, seventeen pairs in GSE23878 from Saudi Arabia, and nineteen pairs in GSE32323 from Japan). The cancer and noncancerous samples were divided into cancer and noncancer groups, and the noncancer group was used as control.

2.2. Identification of DEGs. The selected samples of GSE21510, GSE23878, and GSE32323 were separately analyzed by $\mathrm{R}$ software (https://www.r-project.org). At first, raw data files of the three datasets were downloaded from the GEO database. Then, the Robust Multichip Average algorithm was applied to perform background correction and quantile normalization with the R package Affy $[8,9]$. Next, the probability of genes being differentially expressed between cancer and noncancer groups were calculated by the Linear Models for Microarray Data (LIMMA) package. Finally, the DEGs were selected under corrected $P<0.05$ and |fold change $\mid \geq 2.0$ criteria. The volcano plots of differentially expressed genes were also performed by $\mathrm{R}$ software [10]. The DEGs from each dataset were intersected to identify common DEGs.

2.3. Gene Ontology and Pathway Enrichment Analysis of DEGs. Gene Ontology (GO, http://www.geneontology.org) was a framework for the model of biology that describes the attributes of gene products. It was classified into three aspects: molecular function, biological process, and cellular component [11]. Pathway analysis was a popular method to analyze microarray data in a more detailed, specific way $[12,13]$. DAVID database was a free online bioinformatics resource that aimed to provide functional interpretation of large lists of genes derived from genomic studies [14]. The common DEGs were input in the DAVID database to perform the GO and pathway analysis [15]. $P<0.05$ was considered statistically significant.

2.4. Patients and Tissue Specimens. Tumor tissue samples and individual-matched adjacent mucosa samples were obtained from sixteen patients with colorectal cancer who underwent resection at Xiangya Hospital between 2014 and 2016, and the adjacent mucosa samples were acquired $2-5 \mathrm{~cm}$ away from the tumor. The dissected tissue samples were collected in the operating room and stored immediately in liquid nitrogen. This study was approved by the Institutional Review Board of Department of Clinical Pharmacology, Xiangya Hospital, Central South University (registration number: CTXY-150001-2) and by Chinese Clinical Trial Registry (registration number: ChiCTR-DCD-15006289).

2.5. RNA Isolation, Reverse Transcription, and Real-Time $P C R$. Tissue specimens were grounded and added with TRIzol reagent (Takara). Then the total RNA was isolated, and $1 \mu \mathrm{g}$ of RNA was reverse-transcripted with PrimeScript 1st Strand complementary DNA Synthesis kit (Takara). Realtime PCR assay was performed on ABI 7500 platform. SYBR Premix Dimer Eraser kit (Takara) was used in $20 \mu \mathrm{l}$ reaction volume, and the cycling conditions were as follows: an initial $30 \mathrm{~s}$ denaturation at $95^{\circ} \mathrm{C}$ and 45 cycles $\left(5 \mathrm{~s}\right.$ at $95^{\circ} \mathrm{C}, 30 \mathrm{~s}$ at $55^{\circ} \mathrm{C}$, and $34 \mathrm{~s}$ at $72^{\circ} \mathrm{C}$ ). PPIA and $B 2 M$ genes were set as internal controls. MCM2, RNASEH $2 A$, and TOP2A expression level was detected in sixteen pairs of colorectal cancer and adjacent mucosa samples. The primer sequences were shown in Supplementary Table 1.

2.6. Statistical Analysis. Statistical analyses were performed with SPSS version 18.0 (IBM Corporation) and GraphPad Prism 6.0 software (GraphPad Software Inc.). DEGs were determined using t-statistics from the LIMMA Bioconductor package. The real-time PCR data was analyzed with $2^{-\Delta \mathrm{Ct}}$ and the significance of the difference between the cancer and noncancer groups was evaluated by two-tailed Student's $t$-test. $P$ values less than 0.05 were considered statistically significant.

\section{Results}

3.1. Identification of DEGs. Analysis results showed 1623 upregulated and 1179 downregulated DEGs in GSE21510 (Figure 1(a)), 284 upregulated and 627 downregulated DEGs in GSE23878 (Figure 1(b)), and 717 upregulated and 719 downregulated DEGs (Figure 1(c)) in GSE32323. Then, the DEGs of the three datasets were merged. 463 common DEGs were found in total (Supplementary Table 1), consisting of 181 upregulated and 282 downregulated genes (Figures 1(d)-1(e)).

3.2. GO Term Enrichment Analysis. The 181 upregulated genes were uploaded to the DAVID database for GO analysis. The results showed that upregulated DEGs were significantly enriched in biological process, including 22 DEGs in cell division $(P=1.48 E-11)$ and 19 in mitotic nuclear division $(P=2.06 E-11)$ GO terms. In the cellular component, nucleoplasm $(P=4.95 E-07)$, spindle microtubule $(P=2.49$ $E-06)$, and spindle pole $(P=6.09 E-06)$ were the top three GO terms, and there were 51 DEGs enriched in the nucleoplasm. In molecular function, the top three GO terms were frizzled binding $(P=3.70 E-04)$, protein binding $(P=4.65 E-04)$, and microtubule binding $(P=$ $8.73 E-04)$ (Table 1).

The 282 downregulated DEGs were also imported into the DAVID database. GO analysis results showed that the top three terms in biological process were bicarbonate transport $(P=2.88 E-06)$, negative regulation of growth $(P=5.88 E-06)$, and cellular response to zinc ion $(P=$ $5.88 E-06)$. Extracellular exosome $(P=4.45 E-08)$, extracellular space $(P=2.88 E-06)$, and bicarbonate transport 


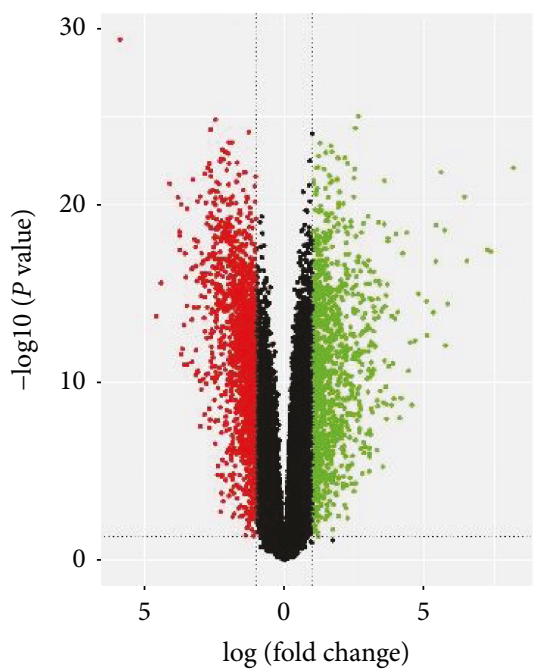

(a)

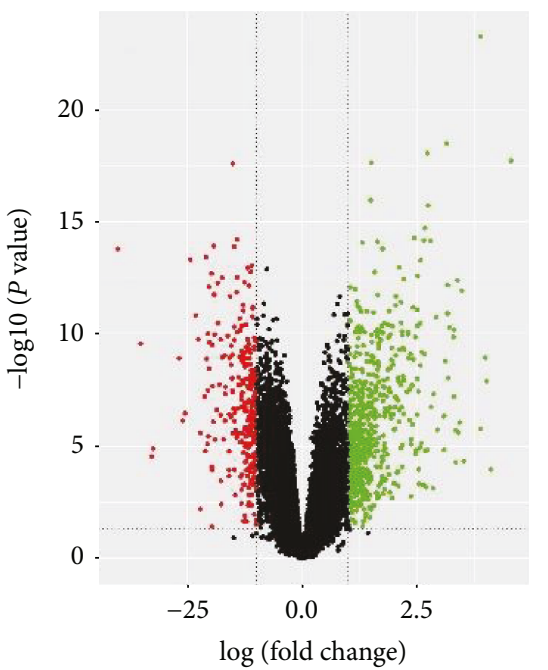

(b)

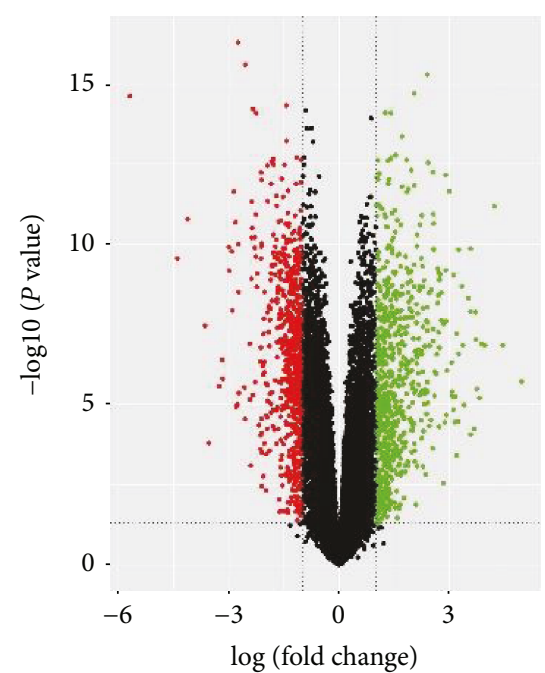

(c)

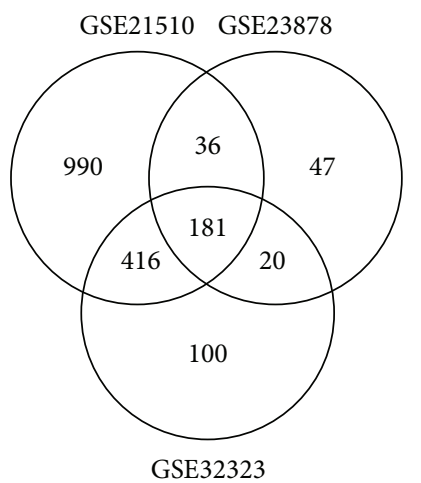

(d)

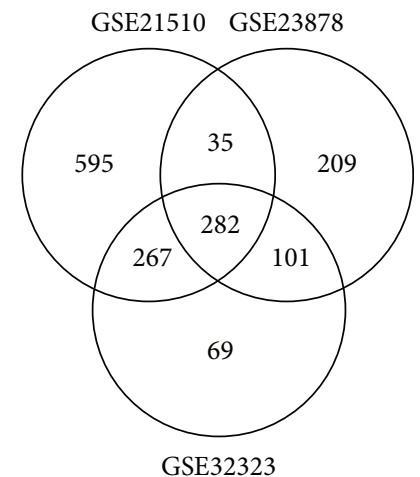

(e)

FIGURE 1: Identification of DEPs and DEGs between tumor and nontumor samples. (a-c) Volcano plot of the differential mRNA expression analysis. $x$-axis: $\log 2$ fold change; $y$-axis: $-\log 10$ (FDR $P$ value) for each probe; vertical dotted lines: fold change $\geq 2$ or $\leq 2$; horizontal dotted line: the significance cutoff (FDR $P$ value $=0.05$ ). (a) There were 2802 genes identified to be differentially expressed in GSE21510, including 1623 upregulated and 1179 downregulated genes. (b) There were 911 genes identified to be differentially expressed in GSE23878, including 284 upregulated and 627 downregulated genes. (c) There were 1436 genes identified to be differentially expressed in GSE32323, including 717 upregulated and 719 downregulated genes. (d-e) Overlap analysis of upregulated genes and downregulated genes between different datasets. (d) A total of 181 genes were significantly upregulated in the three colorectal cancer datasets. (e) A total of 282 genes were significantly downregulated in the three colorectal cancer datasets.

$(P=8.19 E-05)$ in cellular component were the top three GO terms. In molecular function, carbonate dehydratase activity $(P=3.51 E-05)$, chloride channel activity $(P=1.11$ $E-04)$, and oxidoreductase activity $(P=0.0022)$ were the top three GO terms (Table 1).

3.3. KEGG Pathway Analysis. After all the DEGs were input into the DAVID database, KEGG pathway analysis results were also acquired. In Table 2, the most significantly enriched pathway of the upregulated and downregulated pathways was set out.

The upregulated DEGs were significantly enriched in the cell cycle $(P=2.90 E-07)$, p53 signaling pathway $(P=0.028)$, and DNA replication pathways $(P=0.049)$ (Table 2). The downregulated DEGs were enriched in mineral absorption $(P=8.10 E-07)$, nitrogen metabolism $(P=1.57 E-04)$, bile secretion $(P=0.001)$, retinol metabolism $(P=0.005)$, proximal tubule bicarbonate reclamation
$(P=0.007)$, pancreatic secretion $(P=0.020)$, pentose and glucuronate interconversions $(P=0.023)$, renin secretion $(P=0.023)$ pathways, and so forth (Table 2).

3.4. Real-Time PCR Validation of DEGs. To test the DEGs of the analysis, MCM2, RNASEH2A, and TOP $2 A$ were chosen to conduct real-time PCR in sixteen pairs of colorectal cancer and adjacent mucosa samples. MCM2, RNASEH2A, and TOP2A expression level in cancer samples was significantly higher than noncancer samples (MCM2, $P<0.001$, Figure 2(a); RNASEH2A, $P<0.001$, Figure 2(b); TOP2A, $P=0.001$, Figure 2(c)).

\section{Discussion}

Colorectal cancer was a complex disease. To get more information for colorectal cancer occurrence, the gene expression data of fifty-nine paired colorectal cancer and noncancer 
TABLE 1: Go analysis of DEGs between paired tumor and nontumor sample.

\begin{tabular}{|c|c|c|c|c|c|}
\hline Expression & Category & Term/gene function & Count & $\%$ & $P$ value \\
\hline \multirow{15}{*}{ Upregulated } & GOTERM_BP_DIRECT & GO:0051301 cell division & 22 & 8.25 & $1.48 E-11$ \\
\hline & GOTERM_BP_DIRECT & GO:0007067 mitotic nuclear division & 19 & 7.13 & $2.06 E-11$ \\
\hline & GOTERM_BP_DIRECT & GO:0000281 mitotic cytokinesis & 7 & 2.63 & $2.51 E-07$ \\
\hline & GOTERM_BP_DIRECT & GO:0030574 collagen catabolic process & 8 & 3.00 & $2.26 E-06$ \\
\hline & GOTERM_BP_DIRECT & GO:0006260GO:0000082 G1/S transition of mitotic cell cycle & 9 & 3.38 & $5.34 E-06$ \\
\hline & GOTERM_CC_DIRECT & GO:0005654 nucleoplasm & 51 & 19.13 & $4.95 E-07$ \\
\hline & GOTERM_CC_DIRECT & GO:0005876 spindle microtubule & 7 & 2.63 & $2.49 E-06$ \\
\hline & GOTERM_CC_DIRECT & GO:0000922 spindle pole & 9 & 3.38 & $6.09 E-06$ \\
\hline & GOTERM_CC_DIRECT & GO:0005819 spindle & 8 & 3.00 & $1.08 E-04$ \\
\hline & GOTERM_CC_DIRECT & GO:0005578 proteinaceous extracellular matrix & 11 & 4.13 & $1.53 E-04$ \\
\hline & GOTERM_MF_DIRECT & GO:0005109 frizzled binding & 5 & 1.88 & $3.70 E-04$ \\
\hline & GOTERM_MF_DIRECT & GO:0005515 protein binding & 106 & 39.75 & $4.65 E-04$ \\
\hline & GOTERM_MF_DIRECT & GO:0008017 microtubule binding & 9 & 3.38 & $8.73 E-04$ \\
\hline & GOTERM_MF_DIRECT & GO:0019901 protein kinase binding & 12 & 4.50 & $9.80 E-04$ \\
\hline & GOTERM_MF_DIRECT & GO:0008009 chemokine activity & 5 & 1.88 & 0.0012 \\
\hline \multirow{15}{*}{ Downregulated } & GOTERM_BP_DIRECT & GO:0015701 bicarbonate transport & 8 & 2.13 & $2.88 E-06$ \\
\hline & GOTERM_BP_DIRECT & GO:0045926 negative regulation of growth & 6 & 1.60 & $5.88 E-06$ \\
\hline & GOTERM_BP_DIRECT & GO:0071294 cellular response to zinc ion & 6 & 1.60 & $5.88 E-06$ \\
\hline & GOTERM_BP_DIRECT & GO:0006730 one-carbon metabolic process & 6 & 1.60 & $6.33 E-05$ \\
\hline & GOTERM_BP_DIRECT & GO:0007586 digestion & 7 & 1.87 & $2.88 E-04$ \\
\hline & GOTERM_CC_DIRECT & GO:0070062 extracellular exosome & 75 & 20.00 & $4.45 E-08$ \\
\hline & GOTERM_CC_DIRECT & GO:0005615 extracellular space & 42 & 11.20 & $2.88 E-06$ \\
\hline & GOTERM_CC_DIRECT & GO:0031526 brush border membrane & 7 & 1.87 & $8.19 E-05$ \\
\hline & GOTERM_CC_DIRECT & GO:0048471 perinuclear region of cytoplasm & 19 & 5.07 & 0.0035 \\
\hline & GOTERM_CC_DIRECT & GO:0016021 integral component of membrane & 93 & 24.81 & 0.0067 \\
\hline & GOTERM_MF_DIRECT & GO:0004089 carbonate dehydratase activity & 5 & 1.33 & $3.51 E-05$ \\
\hline & GOTERM_MF_DIRECT & GO:0005254 chloride channel activity & 7 & 1.87 & $1.11 E-04$ \\
\hline & GOTERM_MF_DIRECT & GO:0016491 oxidoreductase activity & 10 & 2.67 & 0.0022 \\
\hline & GOTERM_MF_DIRECT & GO:0008201 heparin binding & 8 & 2.13 & 0.0080 \\
\hline & GOTERM_MF_DIRECT & GO:0005179 hormone activity & 6 & 1.60 & 0.0108 \\
\hline
\end{tabular}

tissues was extracted from the GSE21510, GSE23878, and GSE32323 datasets. The fifty-nine paired samples were from two countries: Japan (40 samples from SE21510 and GSE32323) and Saudi Arabia (19 samples from SE32323). The regional divergence of the samples might contribute to finding the common DEGs from two the ethnic groups.

After analysis, 181 upregulated DEGs and 282 downregulated common DEGs were screened. Pathway analyses showed that the upregulated DEGs were mainly involved in cell cycle pathway $(n=11), \mathrm{p} 53$ signaling pathway $(n=4)$, and DNA replication pathway $(n=3)$.

Cell cycle plays a crucial role in tumor evolution and progression, so it acts as an important target for antitumor drugs, such as paclitaxel, vincristine, and Adriamycin. In this study, 3 genes (CDK1, CDK4, and CCNB1) refer to cell cycle pathway were also included in the p53 signaling pathway. CDK1 and CDK4 were key protein kinase for cell cycle control [16, 17]. CCNB1 was also a regulatory protein involved in mitosis [18]. In the p53 signaling pathway, the RRM2 gene was an oncogene that overexpressed in colorectal cancer, with its elevated expression correlated with invasion depth, poorly differentiated type, and tumor node metastasis stage [19]. Transcription factor E2F1 could promote RRM2 expression in colorectal cancer cell lines [20].

DNA replication is an important pathway in carcinogenesis, which ranked the third in upregulated DEGs. Three upregulated DEGs refer to DNA replication were $M C M 2$, RFC3, and RNASEH2A. MCM2 was a member of the MCM family (MCM2-7), and all 6 members of this family could form a hexameric protein complex with each other. This complex worked as a DNA helicase to untie the DNA double helix at the initiation stage of DNA synthesis [21]. MCM2 expression was reported to be associated with colorectal cancer stage and prognosis [22] and used to detect colorectal cancer in stool [23].

Eukaryotic RNase $\mathrm{H} 2$ was a heterotrimeric enzyme formed by RNASEH2A, RNASEH2B, and RNASEH2C. RNA$S E H 2 A$ was a catalytic subunit that could hydrolyze RNA/ DNA hybrid substrate with the structural support from RNASEH $2 B$ and RNASEH2C subunits [24]. It had been 
TABLE 2: Pathway analysis of DEGs between paired tumor and nontumor samples.

\begin{tabular}{|c|c|c|c|c|c|}
\hline Expression & Pathway & Count & Term/gene function & $\%$ & $P$ value \\
\hline \multirow{3}{*}{ Upregulated } & hsa04110: Cell cycle & 11 & $\begin{array}{l}\text { CCNB1, CDK1, CDC6, MAD2L1, TTK, BUB1B, ORC6, } \\
\text { MCM2, PTTG1, CDK4, CDC25B }\end{array}$ & 4.13 & $2.90 E-07$ \\
\hline & hsa04115: p53 signaling pathway & 4 & CCNB1, CDK1, RRM2, CDK4 & 1.50 & 0.028 \\
\hline & hsa03030: DNA replication & 3 & RFC3, MCM2, RNASEH2A & 1.13 & 0.049 \\
\hline \multirow{13}{*}{ Downregulated } & hsa04978: mineral absorption & 9 & $\begin{array}{l}\text { SLC26A3, TRPM6, MT1M, MT2A, CYBRD1, MT1E, } \\
\text { MT1H, MT1X, MT1F }\end{array}$ & 2.40 & $8.10 E-07$ \\
\hline & hsa00910: nitrogen metabolism & 5 & $\mathrm{CA} 12, \mathrm{CA} 7, \mathrm{CA} 4, \mathrm{CA} 2, \mathrm{CA} 1$ & 1.33 & $1.57 E-04$ \\
\hline & hsa04976: bile secretion & 7 & $\begin{array}{l}\text { AQP8, PRKACB, SLC51B, CA2, SLC51A, SLC4A4, } \\
\text { ABCG2 }\end{array}$ & 1.87 & 0.001 \\
\hline & hsa00830: retinol metabolism & 6 & ALDH1A1, ADH1C, DHRS9, ADH1B, UGT2A3, RETSAT & 1.60 & 0.005 \\
\hline & $\begin{array}{l}\text { hsa04964: proximal tubule } \\
\text { Bicarbonate reclamation }\end{array}$ & 4 & CA4, CA2, SLC4A4, PCK1 & 1.07 & 0.007 \\
\hline & hsa04972: pancreatic secretion & 6 & SLC26A3, CLCA1, CLCA4, PLA2G10, CA2, SLC4A4 & 1.60 & 0.020 \\
\hline & $\begin{array}{l}\text { hsa00040: pentose and glucuronate } \\
\text { interconversions }\end{array}$ & 4 & AKR1B10, UGDH, UGT2A3, UGP2 & 1.07 & 0.023 \\
\hline & hsa04924: renin secretion & 5 & CLCA1, CLCA4, GNAI1, PDE3A, PRKACB & 1.33 & 0.023 \\
\hline & $\begin{array}{l}\text { hsa04960: aldosterone-regulated } \\
\text { sodium reabsorption }\end{array}$ & 4 & SGK1, NR3C2, HSD11B2, SCNN1B & 1.07 & 0.028 \\
\hline & hsa04530: tight junction & 7 & $\begin{array}{l}\text { CLDN8, EPB41L3, GNAI1, MYH11, JAM2, CLDN23, } \\
\text { MYL9 }\end{array}$ & 1.87 & 0.028 \\
\hline & hsa05204: chemical carcinogenesis & 5 & NAT2, ADH1C, ADH1B, SULT1A2, UGT2A3 & 1.33 & 0.046 \\
\hline & $\begin{array}{l}\text { hsa04670: leukocyte } \\
\text { transendothelial migration }\end{array}$ & 6 & CLDN8, GNAI1, JAM2, CXCL12, CLDN23, MYL9 & 1.60 & 0.049 \\
\hline & hsa05030: cocaine addiction & 4 & GNAI1, MAOA, PRKACB, FOSB & 1.07 & 0.050 \\
\hline
\end{tabular}

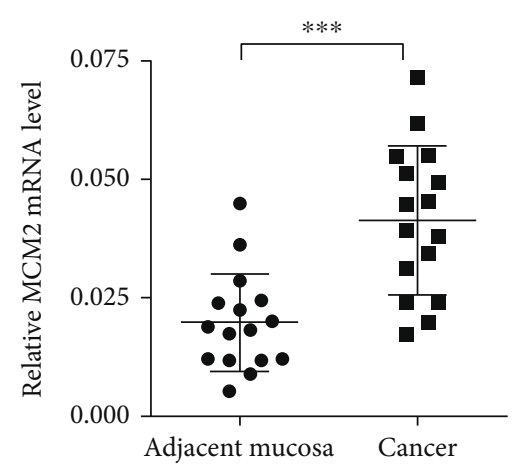

(a)

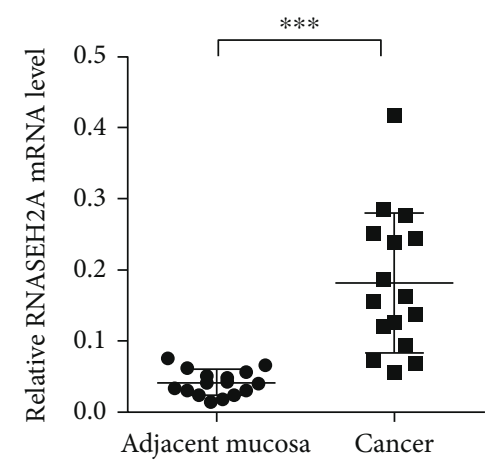

(b)

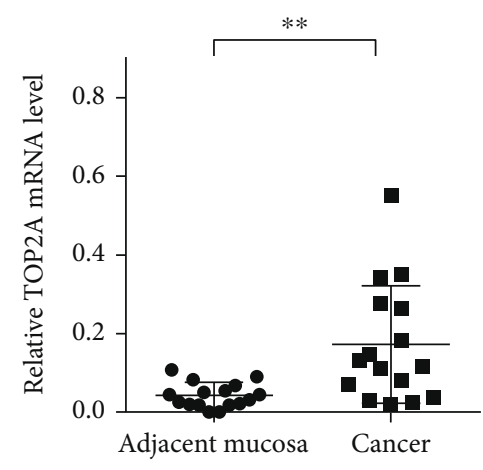

(c)

Figure 2: Validation of the differentially expressed genes. (a) Validation of mRNA expression of MCM2 expression in sixteen colorectal cancer and individual-matched adjacent mucosa samples. MCM2 expression level in colorectal cancer samples was significantly higher than that in adjacent mucosa samples $(P<0.001)$. (b) Validation of mRNA expression of RNASEH2A in sixteen colorectal cancer and individual-matched adjacent mucosa samples. RNASEH2A expression level in colorectal cancer samples was significantly higher than that in adjacent mucosa samples $(P<0.001)$. (c) Validation of mRNA expression of TOP2A in sixteen colorectal cancer and individualmatched adjacent mucosa samples. TOP2A expression level in colorectal cancer samples was significantly higher than that in adjacent mucosa samples $(P=0.001) .{ }^{* *} P<0.01$ and ${ }^{* * *} P<0.001$.

reported that $R N A S E H 2 A$ showed higher expression level in colorectal cancer [25], and this was validated in our research.

TOP $2 A$ was a gene that involves copy number variation and chromosomal instability in many cancers [26-29]. In colorectal cancer, protein expression level of TOP2A was related to aggressive tumor phenotype and advanced tumor stage [30]. In our research, we found that TOP $2 A$ mRNA expression level was upregulated in colorectal cancer.
The downregulated DEGs are mainly involved in mineral absorption, nitrogen metabolism, bile secretion, retinol metabolism, proximal tubule bicarbonate reclamation, pancreatic secretion and so on, which may signal that the tumor cells lose some function for metabolism of the normal colorectal epithelial cell.

In real-time PCR assay, the commonly used internal genes were GAPDH, $\beta$-actin, tubulin, and so forth. However, 
in colorectal cancer tissues, GAPDH was not a good internal control gene since it showed higher transcription level than in normal mucosa, nor was $\beta$-actin $[31,32]$. The combined application of B2M and PPIA were better internal control than others in colorectal cancer [33]. Therefore, $B 2 M$ and PPIA were used as internal control in our study.

In summary, these gene expression profile analysis results suggested 463 candidate biomarkers for early screening and diagnosis of colorectal cancer. Our study confirmed that MCM2, RNASEH2A, and TOP2A were upregulated in colorectal cancer. The protein expression level and functional studies of these markers were warranted to reveal the molecular mechanism of colorectal cancer development.

\section{Conflicts of Interest}

The authors declare that they have no competing interests.

\section{Acknowledgments}

This study was supported by the National Natural Science Foundation of China (Grant no. 81503563), Hainan Provincial Natural Science Foundation of China (Grant no. 310148), Haikou Key Scientific and Technological Project (Grant no. 2012-074).

\section{Supplementary Materials}

Part. 1. Primer sequences for real-time PCR. Part. 2. 181 upregulated and 282 downregulated common differential expressed genes in colorectal cancer samples. (Supplementary Materials)

\section{References}

[1] L. A. Torre, F. Bray, R. L. Siegel, J. Ferlay, J. Lortet-Tieulent, and A. Jemal, "Global cancer statistics, 2012," CA: A Cancer Journal for Clinicians, vol. 65, no. 2, pp. 87-108, 2015.

[2] J. A. Wilschut, J. D. F. Habbema, S. D. Ramsey, R. Boer, C. W. N. Looman, and M. van Ballegooijen, "Increased risk of adenomas in individuals with a family history of colorectal cancer: results of a meta-analysis," Cancer Causes \& Control, vol. 21, no. 12, pp. 2287-2293, 2010.

[3] R. E. Schoen, A. Razzak, K. J. Yu et al., "Incidence and mortality of colorectal cancer in individuals with a family history of colorectal cancer," Gastroenterology, vol. 149, no. 6, pp. 1438-1445.e1, 2015.

[4] J. E. Baars, E. J. Kuipers, M. van Haastert, J. J. Nicolai, A. C. Poen, and C. J. van der Woude, "Age at diagnosis of inflammatory bowel disease influences early development of colorectal cancer in inflammatory bowel disease patients: a nationwide, long-term survey," Journal of Gastroenterology, vol. 47, no. 12, pp. 1308-1322, 2012.

[5] S. P. Cleary, M. Cotterchio, E. Shi, S. Gallinger, and P. Harper, "Cigarette smoking, genetic variants in carcinogenmetabolizing enzymes, and colorectal cancer risk," American Journal of Epidemiology, vol. 172, no. 9, pp. 1000-1014, 2010.

[6] S. Hurley, D. Goldberg, D. O. Nelson et al., "Risk of colorectal cancer associated with active smoking among female teachers," Cancer Causes \& Control, vol. 24, no. 7, pp. 12911304, 2013.
[7] P. Liang and A. B. Pardee, "Timeline: analysing differential gene expression in cancer," Nature Reviews Cancer, vol. 3, no. 11, pp. 869-876, 2003.

[8] R. A. Irizarry, B. M. Bolstad, F. Collin, L. M. Cope, B. Hobbs, and T. P. Speed, "Summaries of Affymetrix GeneChip probe level data," Nucleic Acids Research, vol. 31, no. 4, article e15, 2003.

[9] R. A. Irizarry, B. Hobbs, F. Collin et al., "Exploration, normalization, and summaries of high density oligonucleotide array probe level data," Biostatistics, vol. 4, no. 2, pp. 249-264, 2003.

[10] Y. X. Shi, T. Zhu, T. Zou et al., "Prognostic and predictive values of CDK1 and MAD2L1 in lung adenocarcinoma," Oncotarget, vol. 7, no. 51, pp. 85235-85243, 2016.

[11] T. Torto-Alalibo, E. Purwantini, J. Lomax, J. C. Setubal, B. Mukhopadhyay, and B. M. Tyler, "Genetic resources for advanced biofuel production described with the Gene Ontology," Frontiers in Microbiology, vol. 5, 2014.

[12] C. A. Tilford and N. O. Siemers, "Gene set enrichment analysis," Methods in Molecular Biology, vol. 563, pp. 99-121, 2009.

[13] R. K. Curtis, M. Oresic, and A. Vidal-Puig, "Pathways to the analysis of microarray data," Trends in Biotechnology, vol. 23, no. 8, pp. 429-435, 2005.

[14] B. T. Sherman, D. Huang, Q. Tan et al., "DAVID Knowledgebase: a gene-centered database integrating heterogeneous gene annotation resources to facilitate high-throughput gene functional analysis," BMC Bioinformatics, vol. 8, no. 1, p. 426, 2007.

[15] Y. F. Gao, X. Y. Mao, T. Zhu et al., "COL3A1 and SNAP91: novel glioblastoma markers with diagnostic and prognostic value," Oncotarget, vol. 7, no. 43, pp. 70494-70503, 2016.

[16] G. Banyai, F. Baidi, D. Coudreuse, and Z. Szilagyi, "Cdk1 activity acts as a quantitative platform for coordinating cell cycle progression with periodic transcription," Nature Communications, vol. 7, article 11161, 2016.

[17] M. Malumbres and M. Barbacid, "Cell cycle, CDKs and cancer: a changing paradigm," Nature Reviews Cancer, vol. 9, no. 3, pp. 153-166, 2009.

[18] Y. Fang, H. Yu, X. Liang, J. Xu, and X. Cai, "Chk1-induced CCNB1 overexpression promotes cell proliferation and tumor growth in human colorectal cancer," Cancer Biology \& Therapy, vol. 15, no. 9, pp. 1268-1279, 2014.

[19] A. G. Lu, H. Feng, P. X. Wang, D. P. Han, X. H. Chen, and M. H. Zheng, "Emerging roles of the ribonucleotide reductase M2 in colorectal cancer and ultraviolet-induced DNA damage repair," World Journal of Gastroenterology, vol. 18, no. 34, pp. 4704-4713, 2012.

[20] Z. Fang, C. Gong, H. Liu et al., "E2F1 promote the aggressiveness of human colorectal cancer by activating the ribonucleotide reductase small subunit M2,"Biochemical and Biophysical Research Communications, vol. 464, no. 2, pp. 407-415, 2015.

[21] D. Maiorano, M. Lutzmann, and M. Mechali, "MCM proteins and DNA replication," Current Opinion in Cell Biology, vol. 18, no. 2, pp. 130-136, 2006.

[22] C. Giaginis, M. Georgiadou, K. Dimakopoulou et al., "Clinical significance of MCM-2 and MCM-5 expression in colon cancer: association with clinicopathological parameters and tumor proliferative capacity," Digestive Diseases and Sciences, vol. 54, no. 2, pp. 282-291, 2009.

[23] R. J. Davies, A. Freeman, L. S. Morris et al., "Analysis of minichromosome maintenance proteins as a novel method for 
detection of colorectal cancer in stool," Lancet, vol. 359, no. 9321, pp. 1917-1919, 2002.

[24] H. Chon, A. Vassilev, M. L. DePamphilis et al., "Contributions of the two accessory subunits, RNASEH2B and RNASEH2C, to the activity and properties of the human RNase $\mathrm{H} 2$ complex," Nucleic Acids Research, vol. 37, no. 1, pp. 96-110, 2009.

[25] C. A. Yang, H. Y. Huang, Y. S. Chang, C. L. Lin, I. L. Lai, and J. G. Chang, "DNA-sensing and nuclease gene expressions as markers for colorectal cancer progression," Oncology, vol. 92, no. 2, pp. 115-124, 2017.

[26] T. Chen, Y. Sun, P. Ji, S. Kopetz, and W. Zhang, “Topoisomerase II $\alpha$ in chromosome instability and personalized cancer therapy," Oncogene, vol. 34, no. 31, pp. 4019-4031, 2015.

[27] A. M. Bofin, B. Ytterhus, and B. M. Hagmar, "TOP2A and HER-2 gene amplification in fine needle aspirates from breast carcinomas," Cytopathology, vol. 14, no. 6, pp. 314-319, 2003.

[28] R. Simon, R. Atefy, U. Wagner et al., "HER-2 and TOP2A gene amplification in urinary bladder cancer," Verhandlungen der Deutschen Gesellschaft für Pathologie, vol. 86, pp. 176-183, 2002.

[29] I. M. H. Sønderstrup, S. B. Nygård, T. S. Poulsen et al., "Topoisomerase-1 and -2A gene copy numbers are elevated in mismatch repair-proficient colorectal cancers," Molecular Oncology, vol. 9, no. 6, pp. 1207-1217, 2015.

[30] A. Coss, M. Tosetto, E. J. Fox et al., "Increased topoisomerase II $\alpha$ expression in colorectal cancer is associated with advanced disease and chemotherapeutic resistance via inhibition of apoptosis," Cancer Letters, vol. 276, no. 2, pp. 228-238, 2009.

[31] N. Tsuji, C. Kamagata, M. Furuya et al., "Selection of an internal control gene for quantitation of mRNA in colonic tissues," Anticancer Research, vol. 22, no. 6C, pp. 4173-4178, 2002.

[32] E. Sagynaliev, R. Steinert, G. Nestler, H. Lippert, M. Knoch, and M. A. Reymond, "Web-based data warehouse on gene expression in human colorectal cancer," Proteomics, vol. 5, no. 12, pp. 3066-3078, 2005.

[33] E. A. H. Kheirelseid, K. Chang, J. Newell, M. J. Kerin, and N. Miller, "Identification of endogenous control genes for normalisation of real-time quantitative PCR data in colorectal cancer," BMC Molecular Biology, vol. 11, no. 1, p. 12, 2010. 


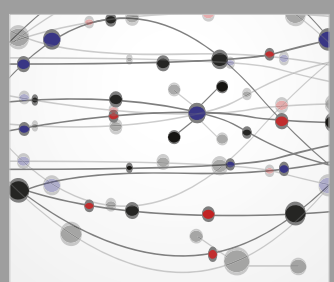

The Scientific World Journal
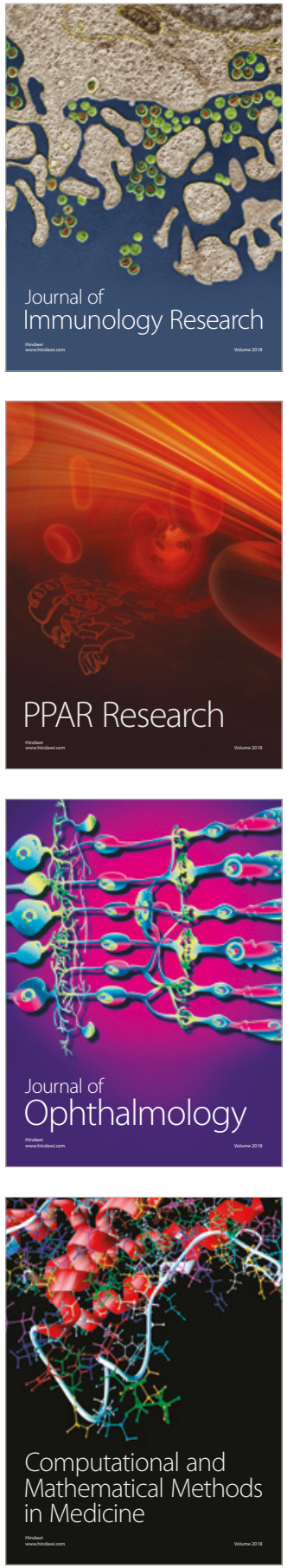

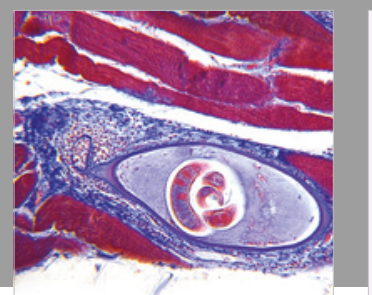

Gastroenterology Research and Practice

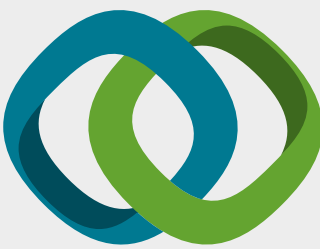

\section{Hindawi}

Submit your manuscripts at

www.hindawi.com
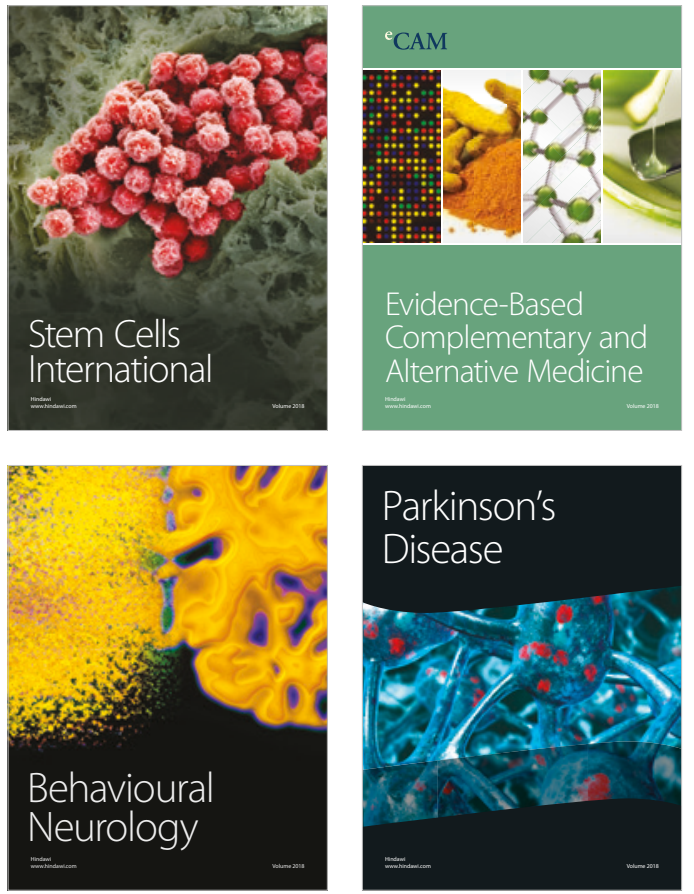

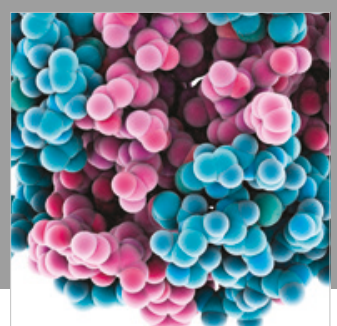

ournal of

Diabetes Research

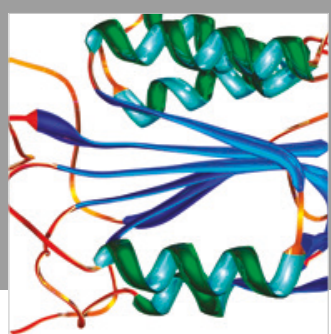

Disease Markers
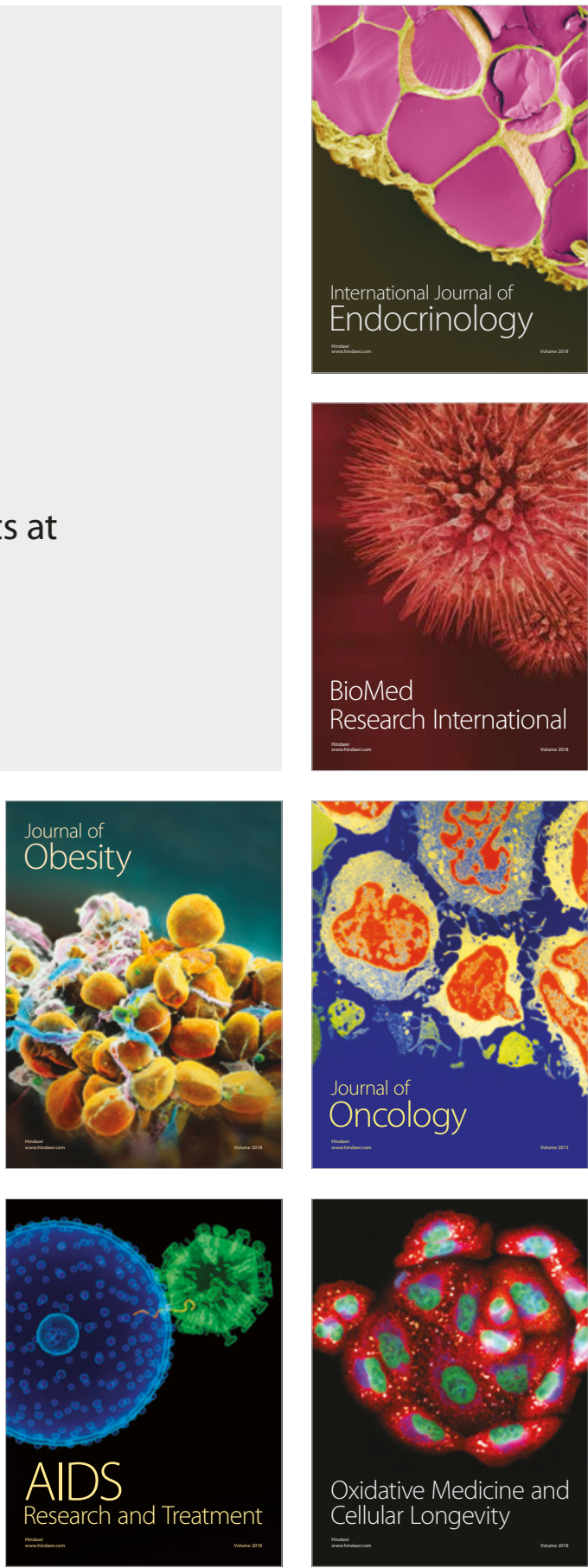